\title{
Replication of genome-wide association studies (GWAS) loci for fasting plasma glucose in African-Americans
}

\author{
E. Ramos • G. Chen • D. Shriner • A. Doumatey • \\ N. P. Gerry • A. Herbert $\cdot$ H. Huang $\cdot$ J. Zhou $•$ \\ M. F. Christman • A. Adeyemo • C. Rotimi
}

Received: 16 July 2010 / Accepted: 8 November 2010 /Published online: 25 December 2010

(C) The Author(s) 2010. This article is published with open access at Springerlink.com

\begin{abstract}
Aims/hypothesis Chronically elevated blood glucose (hyperglycaemia) is the primary indicator of type 2 diabetes, which has a prevalence that varies considerably by ethnicity in the USA, with African-Americans disproportionately affected. Genome-wide association studies (GWASs) have significantly enhanced our understanding of the genetic basis of diabetes and related traits, including fasting plasma glucose (FPG). However, the majority of GWASs have been conducted in populations of European ancestry. Thus, it is important to conduct replication analyses in populations with nonEuropean ancestry to identify shared loci associated with FPG across populations.

Methods We used data collected from non-diabetic unrelated African-American individuals $(n=927)$ who participated in the Howard University Family Study to attempt to replicate previously published GWASs of FPG. Of the 29 single
\end{abstract}

Electronic supplementary material The online version of this article (doi:10.1007/s00125-010-2002-7) contains supplementary material, which is available to authorised users.

E. Ramos $\cdot$ G. Chen $\cdot$ D. Shriner $\cdot$ A. Doumatey $\cdot$ H. Huang $\cdot$

J. Zhou $\cdot$ A. Adeyemo $\cdot$ C. Rotimi $(\triangle)$

Center for Research on Genomics and Global Health, National

Human Genome Research Institute, National Institutes of Health,

12 South Drive, MSC 5635,

Bethesda, MD 20892, USA

e-mail: rotimic@mail.nih.gov

N. P. Gerry • M. F. Christman

Coriell Institute for Medical Research,

Camden, NJ, USA

\section{A. Herbert}

Department of Genetics and Genomics,

Boston University School of Medicine,

Boston, MA, USA nucleotide polymorphisms (SNPs) previously reported, we directly tested 20 in this study. In addition to the direct test, we queried a $500 \mathrm{~kb}$ window centred on all 29 reported SNPs for local replication of additional markers in linkage disequilibrium (LD).

Results Using direct SNP and LD-based comparisons, we replicated multiple SNPs previously associated with FPG and strongly associated with type 2 diabetes in populations with European ancestry. The replicated SNPs included those in or near TCF7L2, SLC30A8, G6PC2, MTNR1B, $D G K B-T M E M 195$ and $G C K R$. We also replicated additional variants in LD with the reported SNPs in ZMAT4 and adjacent to IRSI.

Conclusions/interpretation We identified multiple GWAS variants for FPG in our cohort of African-Americans. Using an LD-based strategy we also identified SNPs not previously reported, demonstrating the utility of using diverse populations for replication analysis.

Keywords African-American - Association - GWAS ·

Replication · Type 2 diabetes

\begin{tabular}{|c|c|}
\hline \multicolumn{2}{|c|}{ Abbreviations } \\
\hline CEU & $\begin{array}{l}\text { Centre d'Etude du Polymorphisme } \\
\text { (Utah residents with northern and western } \\
\text { European ancestry) }\end{array}$ \\
\hline FPG & Fasting plasma glucose \\
\hline GWAS & Genome-wide association study \\
\hline HUFS & Howard University Family Study \\
\hline LD & Linkage disequilibrium \\
\hline MAF & Minor allele frequency \\
\hline MAGIC & $\begin{array}{l}\text { Meta-analyses of Glucose and Insulin-related } \\
\text { traits Consortium }\end{array}$ \\
\hline SNP & Single nucleotide polymorphism \\
\hline YRI & Yoruba in Ibadan, Nigeria \\
\hline
\end{tabular}




\section{Introduction}

Genome-wide association studies (GWASs) have significantly added to our understanding of the genetic basis of type 2 diabetes and related traits, including fasting plasma glucose (FPG), by identifying a number of genes potentially involved in the pathophysiology of this common complex disease [1]. However, the majority of FPG GWASs have been conducted in individuals of European descent, many of which were included in a recent meta-analysis [2]. While this information has laid an important foundation, it is important to investigate whether identified loci transfer across populations with different ancestral backgrounds [3] and whether novel variants could be identified as recently demonstrated in populations of East Asian and Indian backgrounds [4, 5]. Here, we conducted replication of published GWAS results for FPG in African-Americans from the metropolitan area of Washington DC, USA.

\section{Methods}

Ethics statement Ethical approval for the Howard University Family Study (HUFS) was obtained from the Howard University Institutional Review Board and written informed consent was obtained from each participant.

Study design The individuals studied were unrelated nondiabetic participants over the age of 20 years $(n=927)$ enrolled in the HUFS. This population-based study of African-Americans in the Washington, DC metropolitan area has been previously described by Adeyemo et al. [6]. For the present study, participants with $\mathrm{FPG} \geq 7 \mathrm{mmol} / \mathrm{l}$ or who were receiving treatment for diabetes were excluded. Additional characteristics of the cohort can be found in Electronic supplementary material (ESM) Table 1.

Genotyping All 927 DNA samples were prepared and genotyped as described by Adeyemo et al. [6]. Briefly, all samples passed a sample success rate of $95 \%$. Single nucleotide polymorphisms (SNPs) were excluded if they had a success rate of less than $95 \%$ (41,885 SNPs excluded), a minor allele frequency (MAF) $\leq 0.01 \quad(19,154$ SNPs excluded), or had a $p$ value for the Hardy-Weinberg test of equilibrium $<10^{-3}$ (6,317 SNPs excluded). The current analysis focuses on the 808,465 autosomal SNPs that passed these filters. In addition, imputation was performed as reported by Shriner et al. [3]. We successfully imputed $1,506,100$ SNPs using the Yoruba in Ibadan, Nigeria (YRI) reference panel and an additional 52,291 SNPs using the Centre d'Etude du Polymorphisme (Utah residents with northern and western European ancestry) (CEU) reference panel, for a total of $2,366,856$ experimentally determined and imputed SNPs.

Statistical analyses FPG was log-transformed and values greater than \pm 3 SDs from the mean value were winsorised $(n=8)$. All regression models were adjusted for age, sex, BMI and one EIGENSTRAT axis under an additive model. In separate analyses, hypertension was also adjusted for the known association with insulin resistance [7], but the effect was inconsistent, with the magnitude of the $p$ value marginally increasing or decreasing significance for some SNPs (data not shown).

Replication analysis was performed on SNPs identified in GWASs of FPG based on information in the National Human Genome Research Institute's catalogue of published GWASs (www.genome.gov/gwastudies/). The query returned hits indicating reported SNPs, their respective $p$ values and associated genes (ESM Table 2). If multiple studies reported the same SNP, the SNPs with the lowest $p$ value were included in the present study. The returned results included 16 SNPs associated with FPG in the MetaAnalyses of Glucose and Insulin-related traits Consortium (MAGIC) study [2]. The MAGIC SNPs were supplemented by 13 additional SNPs from previously published GWASs (ESM Table 2) for a total of 29 SNPs that we attempted to replicate in our African-American cohort.

Our replication effort occurred in two stages. In the first stage, we attempted to replicate 20 of the 29 exact published SNPs (i.e. direct replication) available in the HUFS dataset. For this stage, SNPs were considered replicated if the same HUFS SNP had a $p$ value $<0.05$. For the second stage, we performed a local replication analysis based on a $500 \mathrm{~kb}$ linkage disequilibrium (LD) block containing a query SNP determined by the SNPs most distant from the query SNP with $r^{2} \geq 0.3$. We used the HapMap CEU LD data (http:/hapmap.ncbi.nlm.nih.gov/ downloads/ld_data/2008-06/00README.TXT) for all SNPs except for rs2166706 where the Gujarati Indians in Houston, Texas, USA (GIH) reference dataset (http:// hapmap.ncbi.nlm.nih.gov/downloads/ld_data/2008-09 phaseIII/00README.txt) was used to match the original reported GWAS population [5]. Second, we estimated the covariance matrix for the block of SNPs using the HUFS genotype data. Third, the covariance matrix was spectrally decomposed and the effective degrees of freedom, $\mathrm{N}_{\text {eff }}$, were estimated using the relationship $\mathrm{N}_{\text {eff }}=\left(\sum_{k=1}^{K} \lambda_{k}\right)^{2} /\left(\sum_{k=1}^{K} \lambda_{k}^{2}\right)$, in which $\lambda_{k}$ is the $k$ th eigenvalue of the $K \times K$ covariance matrix for the $K$ SNPs [8]. Fourth, the nominal significance threshold $\alpha=0.05$ was divided by $\mathrm{N}_{\text {eff. }}$.

Power calculations were carried out using the Quanto software package (Version 1.2.3, http://hydra.usc.edu/gxe/). 
Calculations were based on: continuous outcome; an independent individuals design; and a gene-only hypothesis. An additive inheritance model was applied for varying MAFs. MAFs were calculated based on HUFS data; for SNPs with no associated HUFS data, HapMap- or Perlegenreported MAFs were used. The power for the present study was determined based on reported effect estimates for FPG for each reported MAGIC SNP [2].

\section{Results}

Of the 16 SNPs recently reported in the MAGIC metaanalysis of over 122,000 participants [2], 12 were available for testing in the HUFS dataset (ESM Table 2). We directly replicated three SNPs (rs2191349, rs11558471 and rs4506565) located in or near DGKB-TMEM195, SLC30A8 and TCF7L2 genes respectively (Table 1). We also replicated SNPs from other GWASs for FPG: rs2722425 within ZMAT4 ( $p$ value $=0.024)$ as well as rs625643 $(p$ value $=$ 0.048 ), which is located in a functionally unknown region on chromosome 1 (Table 1). SNPs from the remaining studies that did not directly replicate are not shown. We note that SNPs in C2CD4B, FADS1, GCK and G6PC2 from the MAGIC study and IRS1, PDE4B, and ATP8B4 from other
GWASs were not directly compared in the HUFS dataset owing to quality-control filters or lack of genotyping or imputation data.

We also analysed SNPs that were in LD $\left(r^{2} \geq 0.3\right)$ with each discovery SNP (ESM Fig. 1). This replication strategy, which queried a $500 \mathrm{~kb}$ window centred on the index SNP, yielded a total of 317 SNPs located in or near nine different genes or unknown gene region (G6PC2, GCKR, MTNR1B, DGKB-TMEM195, TCF7L2, SLC30A8, AK024684, ZMAT4 and IRS1). Thirty-eight SNPs distributed across all nine gene regions of the 317 SNPs tested were significantly associated with FPG after Bonferroni correction for multiple comparisons (Table 2).

Based on reported effect sizes of the 14 MAGIC loci (excluding TCF7L2 and SLC30A8), the power was calculated for each SNP using the African-American MAFs where available (ESM Table 3). The estimated power for this study ranged from a low of 0.25 to a high of 0.99 . The SNPs in the four genes most strongly powered (i.e. $>90 \%$ power) in this study were either directly replicated $(D G K B$ TMEM195) or locally replicated (G6PC2, MTNR1B and $G C K R)$ with markers in moderate LD $\left(r^{2} \geq 0.4\right)$. The effect sizes for SNPs rs4506565 and rs11558471 (previously reported loci TCF7L2 and SLC30A8, respectively) were not reported in the MAGIC study.

Table 1 SNPs that were reported in the MAGIC study and other GWASs of FPG that were directly analysed for replication in a cohort of African-Americans (the HUFS)

\begin{tabular}{|c|c|c|c|c|c|c|c|}
\hline \multirow[t]{2}{*}{ SNP } & \multirow[t]{2}{*}{ Nearest gene } & \multirow[t]{2}{*}{ Region } & \multirow[t]{2}{*}{ Position } & \multirow[t]{2}{*}{ Reported $p$ value } & \multicolumn{3}{|l|}{ HUFS } \\
\hline & & & & & $\begin{array}{l}\text { Allele } \\
\text { (effect/other) }\end{array}$ & $\beta$ & $p$ value \\
\hline \multicolumn{8}{|l|}{ HUFS } \\
\hline rs 340874 & PROX1 & $1 \mathrm{q} 41$ & 212225879 & $7.1 \times 10^{-8}$ & $\mathrm{C} / \mathrm{T}$ & -0.0124 & 0.191 \\
\hline rs780094 & $G C K R$ & $2 \mathrm{p} 23.2$ & 27594741 & $2.5 \times 10^{-12}$ & $\mathrm{~T} / \mathrm{C}$ & 0.0034 & $0.66^{\mathrm{a}}$ \\
\hline rs11708067 & $A D C Y 5$ & $3 q 13.33$ & 124548468 & $8.7 \times 10^{-9}$ & $\mathrm{G} / \mathrm{A}$ & -0.0028 & $0.73^{\mathrm{a}}$ \\
\hline rs11920090 & $S L C 2 A 2$ & $3 \mathrm{q} 26.2$ & 172200215 & $1.9 \times 10^{-6}$ & $\mathrm{~A} / \mathrm{T}$ & 0.0001 & 1.0 \\
\hline rs2191349 & DGKB-TMEM195 & $7 \mathrm{p} 21.2$ & 15030834 & $7.8 \times 10^{-17}$ & $\mathrm{G} / \mathrm{T}$ & -0.0136 & $0.023^{\mathrm{b}}$ \\
\hline rs 11558471 & $S L C 30 A 8$ & $8 \mathrm{q} 24.11$ & 118254914 & $2.6 \times 10^{-11}$ & $\mathrm{G} / \mathrm{A}$ & -0.0255 & $0.0074^{\mathrm{b}}$ \\
\hline rs7034200 & GLIS3 & $9 \mathrm{p} 24.2$ & 4279050 & $1.2 \times 10^{-4}$ & $\mathrm{C} / \mathrm{A}$ & 0.0056 & 0.35 \\
\hline rs 10885122 & $A D R A 2 A$ & $10 \mathrm{q} 25.2$ & 113032083 & $8.4 \times 10^{-11}$ & $\mathrm{G} / \mathrm{T}$ & 0.0079 & 0.2 \\
\hline rs 4506565 & $T C F 7 L 2$ & $10 \mathrm{q} 25.2$ & 114746031 & $1.2 \times 10^{-8}$ & $\mathrm{~T} / \mathrm{A}$ & -0.0117 & $0.036^{\mathrm{a}, \mathrm{b}}$ \\
\hline rs 10830963 & MTNR1B & $11 \mathrm{q} 21$ & 92348358 & $1.2 \times 10^{-68}$ & $\mathrm{G} / \mathrm{C}$ & -0.0046 & $0.67^{\mathrm{a}}$ \\
\hline rs11605924 & $C R Y 2$ & $11 \mathrm{p} 11.2$ & 45829667 & $1.5 \times 10^{-9}$ & $\mathrm{C} / \mathrm{A}$ & 0.0048 & 0.57 \\
\hline rs7944584 & $M A D D$ & $11 \mathrm{p} 11.2$ & 47292896 & $1.5 \times 10^{-9}$ & $\mathrm{~T} / \mathrm{A}$ & 0.0026 & $0.84^{\mathrm{a}}$ \\
\hline \multicolumn{8}{|c|}{ From other FPG GWASs } \\
\hline rs625643 & $A K 024684$ & $1 \mathrm{p} 32.3$ & 54409755 & $4.0 \times 10^{-3}$ & $\mathrm{~T} / \mathrm{C}$ & 0.01141 & $0.05^{\mathrm{a}, \mathrm{b}}$ \\
\hline rs2722425 & ZMAT4 & $8 \mathrm{p} 11.21$ & 40603396 & $9.0 \times 10^{-6}$ & $\mathrm{~T} / \mathrm{C}$ & 0.01326 & $0.024^{\mathrm{b}}$ \\
\hline
\end{tabular}

${ }^{\text {a }}$ Genotyped HUFS SNPs (all other SNPs were imputed)

${ }^{\mathrm{b}}$ SNPs from reported studies that are also statistically significant $(p<0.05)$ in the HUFS dataset 
Table 2 Significant SNPs and their effects $(\beta)$ after Bonferroni correction that were in LD $\left(r^{2} \geq 0.3\right)$ with reported SNPs from the MAGIC study and other GWASs of FPG

\begin{tabular}{|c|c|c|c|c|c|c|c|c|c|c|}
\hline \multirow[t]{2}{*}{ Region } & \multirow[t]{2}{*}{ Nearest gene } & \multicolumn{2}{|l|}{ Reported } & \multirow[t]{2}{*}{$\mathrm{SNP}$ in LD } & \multicolumn{6}{|l|}{ Study results } \\
\hline & & SNP & $p$ value & & $\begin{array}{l}\text { Allele } \\
\text { (effect/other) }\end{array}$ & $\beta$ & $r^{2}$ & $p$ value & $d f^{a}$ & $\begin{array}{l}\text { Corrected } \\
p \text { value }\end{array}$ \\
\hline \multicolumn{11}{|l|}{ HUFS } \\
\hline $2 \mathrm{q} 31.1$ & G6PC2 & rs560887 & $4.4 \times 10^{-35}$ & rs575671 & $\mathrm{A} / \mathrm{G}$ & -0.0185 & 0.50 & 0.003 & 2.76 & 0.01 \\
\hline \multirow[t]{5}{*}{$2 \mathrm{p} 23.2$} & $G C K R$ & rs780094 & $2.5 \times 10^{-12}$ & rs780100 & $\mathrm{G} / \mathrm{T}$ & 0.0142 & 0.46 & 0.020 & 2.55 & 0.05 \\
\hline & & & & rs704791 & $\mathrm{T} / \mathrm{C}$ & 0.0142 & 0.46 & 0.020 & 2.55 & 0.05 \\
\hline & & & & rs780102 & $\mathrm{T} / \mathrm{C}$ & 0.0142 & 0.46 & 0.020 & 2.55 & 0.05 \\
\hline & & & & rs704795 & $\mathrm{G} / \mathrm{A}$ & 0.0154 & 0.46 & 0.011 & 2.55 & 0.03 \\
\hline & & & & rs 10208529 & $\mathrm{~T} / \mathrm{A}$ & -0.0198 & 0.54 & 0.021 & 2.55 & 0.05 \\
\hline \multirow[t]{9}{*}{$7 \mathrm{p} 21.2$} & DGKB-TMEM195 & rs2191349 & $7.8 \times 10^{-17}$ & rs 2215383 & $\mathrm{~T} / \mathrm{C}$ & -0.0175 & 1.0 & 0.003 & 2.38 & 0.007 \\
\hline & & & & rs 10244051 & $\mathrm{~T} / \mathrm{G}$ & -0.0163 & 1.0 & 0.004 & 2.38 & 0.01 \\
\hline & & & & rs 10258074 & $\mathrm{~A} / \mathrm{T}$ & -0.0163 & 0.97 & 0.004 & 2.38 & 0.01 \\
\hline & & & & rs2191348 & $\mathrm{G} / \mathrm{T}$ & -0.0164 & 1.0 & 0.004 & 2.38 & 0.009 \\
\hline & & & & rs6947830 & $\mathrm{G} / \mathrm{A}$ & -0.0131 & 1.0 & 0.022 & 2.38 & $0.05^{\mathrm{b}}$ \\
\hline & & & & rs4719433 & $\mathrm{T} / \mathrm{C}$ & -0.0131 & 1.0 & 0.022 & 2.38 & $0.05^{\mathrm{b}}$ \\
\hline & & & & rs 1974620 & $\mathrm{C} / \mathrm{T}$ & -0.0147 & 0.97 & 0.011 & 2.38 & $0.03^{\mathrm{b}}$ \\
\hline & & & & rs1558317 & $\mathrm{T} / \mathrm{A}$ & -0.0147 & 0.96 & 0.011 & 2.38 & 0.03 \\
\hline & & & & rs 1558318 & $\mathrm{~A} / \mathrm{T}$ & -0.0147 & 0.97 & 0.011 & 2.38 & 0.03 \\
\hline $8 \mathrm{q} 24.11$ & $S L C 30 A 8$ & rs 11558471 & $2.6 \times 10^{-11}$ & rs 11774700 & $\mathrm{C} / \mathrm{T}$ & -0.0249 & 0.78 & 0.004 & 1.74 & 0.006 \\
\hline $10 \mathrm{q} 25.2$ & $T C F 7 L 2$ & rs 4506565 & $1.2 \times 10^{-8}$ & rs7901695 & $\mathrm{C} / \mathrm{T}$ & -0.0127 & 0.88 & 0.022 & 1.72 & 0.04 \\
\hline \multirow[t]{7}{*}{$11 q 21$} & MTNRIB & rs 10830963 & $1.2 \times 10^{-68}$ & rs1447350 & $\mathrm{C} / \mathrm{G}$ & -0.0141 & 0.43 & 0.014 & 2.46 & 0.03 \\
\hline & & & & rs1447351 & $\mathrm{A} / \mathrm{G}$ & -0.0131 & 0.43 & 0.022 & 2.46 & 0.05 \\
\hline & & & & rs4611171 & $\mathrm{T} / \mathrm{G}$ & -0.0131 & 0.42 & 0.022 & 2.46 & $0.05^{\mathrm{b}}$ \\
\hline & & & & rs 1597023 & G/A & -0.0131 & 0.42 & 0.022 & 2.46 & 0.05 \\
\hline & & & & rs4406791 & $\mathrm{A} / \mathrm{C}$ & -0.0131 & 0.42 & 0.022 & 2.46 & 0.05 \\
\hline & & & & rs1447352 & $\mathrm{G} / \mathrm{A}$ & -0.0131 & 0.43 & 0.022 & 2.46 & 0.05 \\
\hline & & & & rs9666752 & $\mathrm{G} / \mathrm{A}$ & -0.0148 & 0.36 & 0.009 & 2.46 & 0.02 \\
\hline \multicolumn{11}{|c|}{ From other FPG GWASs } \\
\hline $1 \mathrm{p} 32.1$ & $A K 024684$ & rs625643 & $4 \times 10^{-3}$ & rs671431 & $\mathrm{G} / \mathrm{C}$ & 0.0143 & 0.62 & 0.022 & 1.66 & 0.04 \\
\hline \multirow[t]{10}{*}{$2 \mathrm{q} 36.1$} & IRSI & rs2943641 & $5 \times 10^{-2}$ & rs6713510 & $\mathrm{A} / \mathrm{T}$ & 0.0169 & 0.43 & 0.005 & 5.13 & 0.03 \\
\hline & & & & rs4072096 & $\mathrm{G} / \mathrm{A}$ & 0.0171 & 0.43 & 0.005 & 5.13 & 0.03 \\
\hline & & & & rs4645008 & $\mathrm{A} / \mathrm{C}$ & 0.0181 & 0.43 & 0.003 & 5.13 & 0.02 \\
\hline & & & & rs2943644 & $\mathrm{T} / \mathrm{C}$ & 0.0181 & 0.41 & 0.003 & 5.13 & 0.02 \\
\hline & & & & rs2943633 & $\mathrm{C} / \mathrm{T}$ & 0.0167 & 0.45 & 0.005 & 5.13 & 0.03 \\
\hline & & & & rs2972150 & $\mathrm{C} / \mathrm{A}$ & 0.0161 & 0.45 & 0.007 & 5.13 & 0.04 \\
\hline & & & & rs2943636 & $\mathrm{C} / \mathrm{T}$ & 0.0165 & 0.45 & 0.006 & 5.13 & 0.03 \\
\hline & & & & rs2972149 & $\mathrm{A} / \mathrm{G}$ & 0.0201 & 0.50 & 0.0007 & 5.13 & $0.004^{\mathrm{b}}$ \\
\hline & & & & rs 1515114 & $\mathrm{~A} / \mathrm{T}$ & 0.0196 & 0.60 & 0.0009 & 5.13 & $0.005^{\mathrm{b}}$ \\
\hline & & & & rs2722429 & $\mathrm{G} / \mathrm{A}$ & 0.0186 & 0.60 & 0.001 & 5.13 & 0.006 \\
\hline \multirow[t]{3}{*}{$8 p 11.21$} & ZMAT4 & rs 2722425 & $9 \times 10^{-6}$ & rs 1823643 & $\mathrm{~T} / \mathrm{C}$ & 0.0192 & 0.89 & 0.002 & 1.53 & 0.007 \\
\hline & & & & & $\mathrm{C} / \mathrm{A}$ & 0.0145 & 0.65 & 0.021 & 1.53 & $0.03^{\mathrm{b}}$ \\
\hline & & & & & $\mathrm{C} / \mathrm{T}$ & 0.0146 & 0.68 & 0.018 & 1.53 & 0.03 \\
\hline
\end{tabular}

${ }^{\mathrm{a}} d f$ calculated from covariance matrix of SNPs in LD $\left(r^{2} \geq 0.3\right)$ with query SNP in $500 \mathrm{~kb}$ window; $d f$ were used for Bonferroni corrections

${ }^{\mathrm{b}}$ Genotyped HUFS SNPs (all other SNPs were imputed) 


\section{Discussion}

Chronically elevated FPG is a primary indicator of diabetes, making it an important barometer of the progression of impaired glucose metabolism. In this paper, we attempted to replicate, in nearly 1,000 African-Americans, significant GWAS loci for FPG in populations of predominantly European ancestry. In light of well-reported increased genetic diversity in populations with African ancestry [9, 10], our replication strategy not only focused on the reported SNPs but also included querying variants in LD with the reported SNPs.

We focused our replication analysis on the MAGIC study of over 122,000 participants to identify FPGassociated SNPs shared across ethnically diverse populations. In addition, we included SNPs from prior GWASs of FPG to add breadth to our replication pool, keeping in mind that potential differences in susceptibility loci between populations may exist [11]. Of the $12 \mathrm{SNPs}$ reported by the MAGIC study that were directly testable in our AfricanAmerican cohort, we replicated three SNPs within $D G K B$ TMEM195, TCF7L2 and SLC30A8. We also replicated SNPs in ZMAT4, which encodes a zinc finger, matrin type 4 protein identified in previous GWASs but not replicated in the MAGIC meta-analysis. Using the local (LD-based) replication strategy, we replicated additional SNPs in or near previously reported genes, including the insulin receptor substrate 1 gene.

Interestingly, comparison of the LD structure in HUFS to HapMap reference samples CEU and YRI supports the utility of African-American population samples in refining association loci. For example, the covariance matrix generated for the local replication of rs625643 spans $40 \mathrm{~kb}$ and includes 16 SNPs. In HapMap CEU, nearly the entire region is in moderate LD, whereas in HapMap YRI two distinct LD blocks are observed (ESM Fig. 2) and lower (on average) $r^{2}$ values are observed between rs625643 and downstream SNPs (0.78 for CEU and 0.5 for YRI). As expected, African-American samples (i.e. HapMap African Ancestry in Southwest, USA [ASW] and this study HUFS) show an LD structure intermediate to CEU and YRI (ESM Fig. 3 and ESM Fig. 2). Furthermore, given the association signals in HUFS, a case can be made for a narrowing of the region of interest from $40 \mathrm{~kb}$ to $3 \mathrm{~kb}$ between the locally replicated SNP rs671431 and the original discovery SNP (ESM Fig. 3).

We acknowledge the fluid interpretation of $r^{2}$ values within the context of establishing variants in $\mathrm{LD}$ with each other as well as the concern of being overly conservative in our correction for multiple comparisons. At an $r^{2} \geq 0.3$, we attempted to first capture a significant portion of SNPs in LD within the reference sample while maintaining confidence in the ability of related SNPs to serve as proxies [12]. In addition, a blanket search window of $500 \mathrm{~kb}$ would allow for capture of some unique characteristics of LD and long-range LD associated with admixed populations such as African-Americans [13]. To address the burden of potentially overcorrecting for multiple comparisons, our Bonferroni correction strategy was based on the estimation of effective degrees of freedom [8], which provides an analysis of covariance among HUFS SNPs in the extracted LD block that was based on CEU HapMap samples. We feel this approach better describes the relationship of SNPs in LD within the queried window instead of assuming the very conservative approach of independent effects for all tested SNPs.

We also acknowledge the limitation of our study of about 1,000 participants to detect some of the very small effect sizes reported by the MAGIC study, which included more than 122,000 participants. However, this study had over $80 \%$ power to replicate similar effect sizes for 10 of the 14 SNPs reported by the MAGIC study (ESM Table 3); this is evident in this study's ability to replicate several of the published GWAS variants for FPG. We caution that lack of replication in this study may be due to limited sample size, differences in effect sizes calculated for SNPs and difference in allele frequency between populations of European and African ancestries.

The need for understanding differential susceptibility to diseases at the population level makes the identification of risk factors for diabetes and its indicators, including FPG, particularly important in the African-American community and other ethnic groups, given their disproportionate rate of morbidity and mortality from diabetes and associated complications. Unfortunately and for multiple reasons, the majority of GWASs aimed at identifying genetic variants associated with FPG and diabetes have so far focused predominantly on individuals of European ancestry. While the results from these studies provided tremendous insight into the genetic architecture of the disease, recent studies of non-European populations have shown utility in expanding the breadth of populations studied. Specifically, studies in East Asians allowed for a 'wider net' to be cast in the identification of type 2 diabetes susceptibility variants [4, 11]. The present study's focus on individuals self-identified as African-Americans not only widens the net but also underscores the need for directed investigation of underrepresented populations.

Acknowledgements The HUFS was supported by National Institutes of Health grants S06GM008016-320107 to C. Rotimi and S06GM008016-380111 to A. Adeyemo. We thank the participants of the study, for which enrolment was carried out at the Howard University General Clinical Research Center, supported by National Institutes of Health grant 2M01RR010284. The contents of this publication are solely the responsibility of the authors and do not necessarily represent the official view of the National Institutes of Health. This research was supported in part by the Intramural Research Program of the Center for 
Research on Genomics and Global Health, which is supported by the National Human Genome Research Institute, the National Institute of Diabetes and Digestive and Kidney Diseases, the Center for Information Technology and the Office of the Director at the National Institutes of Health (Z01HG200362). Genotyping support was provided by the Coriell Institute for Medical Research.

Duality of interest The authors declare that there is no duality of interest associated with this manuscript.

Open Access This article is distributed under the terms of the Creative Commons Attribution Noncommercial License which permits any noncommercial use, distribution, and reproduction in any medium, provided the original author(s) and source are credited.

\section{References}

1. O'Rahilly S (2009) Human genetics illuminates the paths to metabolic disease. Nature 462:307-314

2. Dupuis J, Langenberg C, Prokopenko I et al (2010) New genetic loci implicated in fasting glucose homeostasis and their impact on type 2 diabetes risk. Nat Genet 42:105-116

3. Shriner D, Adeyemo A, Gerry NP et al (2009) Transferability and fine-mapping of genome-wide associated loci for adult height across human populations. PLoS One 4:e8398
4. McCarthy MI (2008) Casting a wider net for diabetes susceptibility genes. Nat Genet 40:1039-1040

5. Chambers JC, Zhang W, Zabaneh D et al (2009) Common genetic variation near melatonin receptor MTNR1B contributes to raised plasma glucose and increased risk of type 2 diabetes among Indian Asians and European Caucasians. Diabetes 58:2703-2708

6. Adeyemo A, Gerry N, Chen G et al (2009) A genome-wide association study of hypertension and blood pressure in African Americans. PLoS Genet 5:e1000564

7. Reaven GM (1991) Insulin resistance, hyperinsulinemia, hypertriglyceridemia, and hypertension. Parallels between human disease and rodent models. Diabetes Care 14:195-202

8. Bretherton CS, Widmann M, Dymnikov VP, Wallace JM, Blade I (1999) The effective number of spatial degrees of freedom of a time-varying field. J Climate 12:1990-2009

9. Schuster SC, Miller W, Ratan A et al (2010) Complete Khoisan and Bantu genomes from southern Africa. Nature 463:943-947

10. Tishkoff SA, Reed FA, Friedlaender FR et al (2009) The genetic structure and history of Africans and African Americans. Science 324:1035-1044

11. Unoki H, Takahashi A, Kawaguchi $\mathrm{T}$ et al (2008) SNPs in KCNQ1 are associated with susceptibility to type 2 diabetes in East Asian and European populations. Nat Genet 40:1098-1102

12. Ardlie KG, Kruglyak L, Seielstad M (2002) Patterns of linkage disequilibrium in the human genome. Nat Rev Genet 3:299-309

13. Evans DM, Cardon LR (2005) A comparison of linkage disequilibrium patterns and estimated population recombination rates across multiple populations. Am J Hum Genet 76:681687 